\title{
Diffraction microscopy - towards an ultra-high-resolution microscope
}

\author{
T. Beetz*, Y. Zhu* \\ * Center for Functional Nanomaterial, Brookhaven National Laboratory, Upton, NY 11973-5000
}

During the past years, a tremendous effort has been put into pushing the resolution of electron microscopes towards and beyond $1 \AA$. This effort has been fueled by the successful development and implementation of aberration correctors to improve the performance of the electron microscope [1, 2]. Unfortunately, this has also increased the stability requirements on microscope components such as stage and high tension among others. In addition, sophisticated and expensive laboratory environments are required for the high-end microscopes to perform at the highest resolution, requiring substantial investment.

Diffraction microscopy offers an alternative to reach sub- $\AA$ resolution with the benefit that no special microscope or environment is required. In diffraction microscopy, the diffraction pattern of a coherently illuminated object is recorded at at least twice the Nyquist frequency and the challenge is to reconstruct the object using only the diffraction pattern [3-5]. Since recording the diffraction pattern is an intensity measurement, the phases are lost. Iterative algorithm can be used to recover the phases and therefore reconstructing the scattering object $[6,7]$. This extensive post-experiment computing necessary to recover the scattering object is both advantageous and worthwhile. One advantage, for example, is that spatial frequencies beyond the information limit of the microscope can easily be recorded by recording the diffraction data. In addition, small sample drift or vibrations are not as critical as in electron microscopy since a small shift adds a constant phase to the diffraction pattern which is canceled out in the intensity recording.

While the potential of electron diffraction microscopy for performing ultra-high-resolution imaging is enormous, little has been done to fully exploit this potential. For example, questions remain regarding the limits of diffraction microscopy, sample suitability, and microscope conditions. Samples should ideally be limited in size and non-periodic to take advantage of the technique. The intensity distribution in the diffraction pattern from such an object is then not concentrated in the Bragg spots but rather diffusively scattered over the whole diffraction pattern. One type of object that is ideal then is small nanoparticles. Recent experiments have used amorphous carbon as a support film [8]. However, the support film has its own internal structure which will give rise to distinctive speckle in the diffraction pattern. This is problematic because the support film must be included in the reconstruction and the speckle is difficult to remove. Our investigations using both a JEOL 2200FS (equipped with an in-column energy filter and image plates) and computer simulations show that errors in the reconstruction emerge when small particles (less than a few hundred atoms) are involved. In order to address the shortcomings induced by using a support film, we have conducted experiments where the nanoparticles are attached to a periodic structure (for example a multi-walled carbon nanotube). Since the nanotube has a well-defined structure giving rise to strong spots in the diffraction pattern, it can be taken out during the reconstruction, leaving only the nanoparticle as the reconstructed object. We will also present experimental results supported by computer simulations to show the validity of using nanotubes as a support structure for a nanoparticle. Finally, we further investigate the limits of diffraction microscopy to determine what kind of sensitivity is needed to detect just a few atoms attached to a nanotube as well as detecting defects in nanotubes. 
References

[1] Haider, M., et al., Ultramicroscopy, 1998. 75(1): p. 53-60.

[2] Batson, P.E., N. Dellby, and O.L. Krivanek, Nature (UK), 2002. 418(6898): p. 617-620.

[3] Miao, J.W., et al., Nature (UK), 1999. 400(6742): p. 342-344.

[4] Sayre, D., Acta Crystallographica, 1952. 5: p. 843.

[5] Zuo, J.M., et al., Science, 2003. 300(5624): p. 1419-1421.

[6] Fienup, J.R., Opt. Lett., 1978. 3(1): p. 27-29.

[7] Elser, V., J. Opt. Soc. Am. A-Opt. Image Sci. Vis., 2003. 20(1): p. 40-55.

[8] Wu, J.S., U. Weierstall, and J.C.H. Spence, Nature Materials, 2005. 4(12): p. 912-916.

[9] This work is funded by the U.S. Department of Energy under contract DE-AC02-98CH10886.

a)

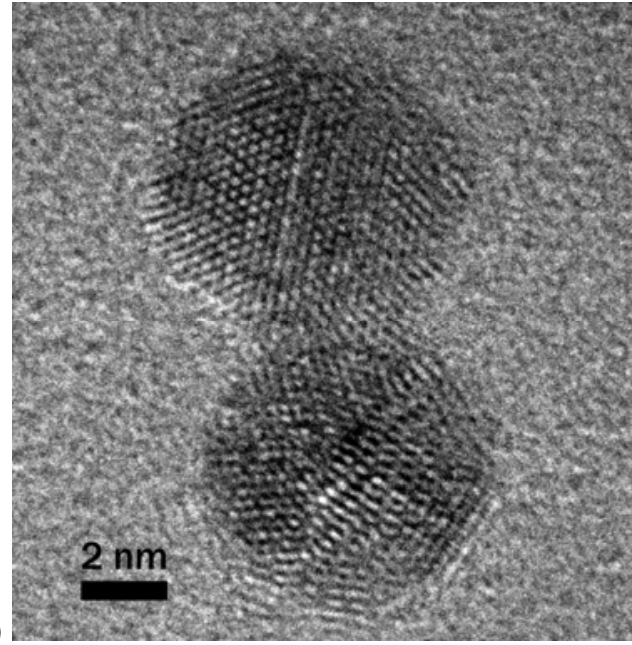

c)

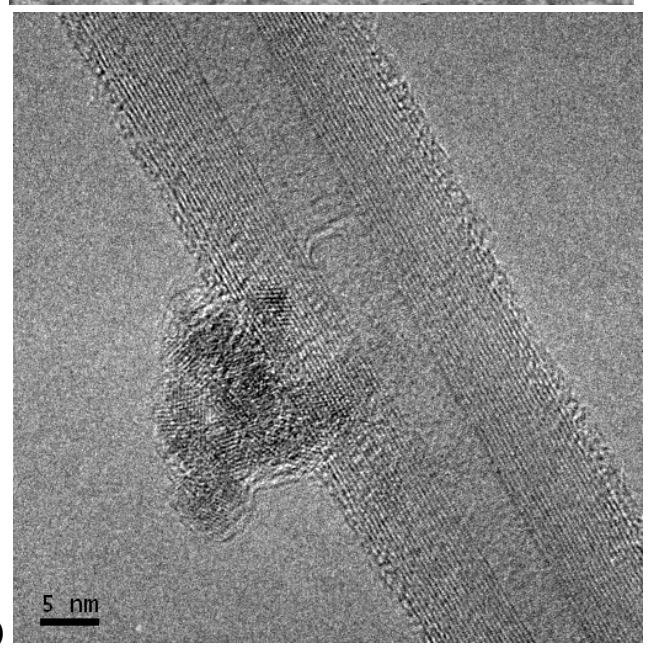

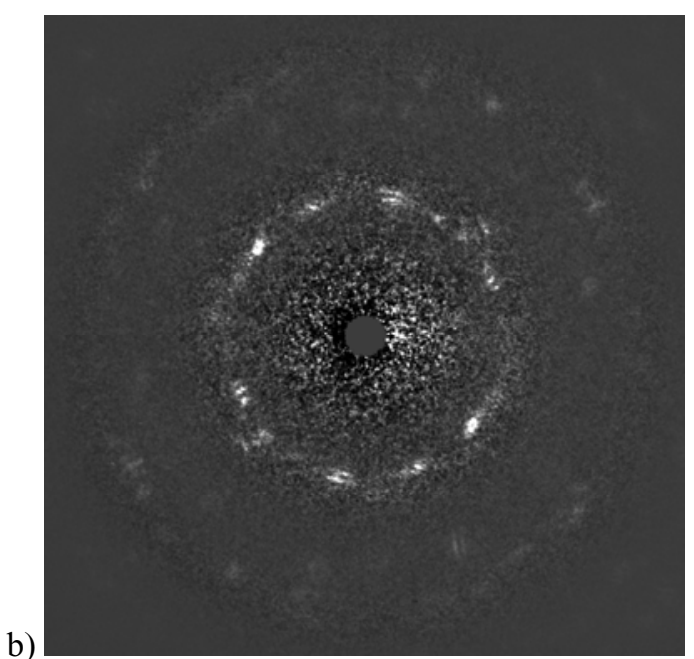

b)

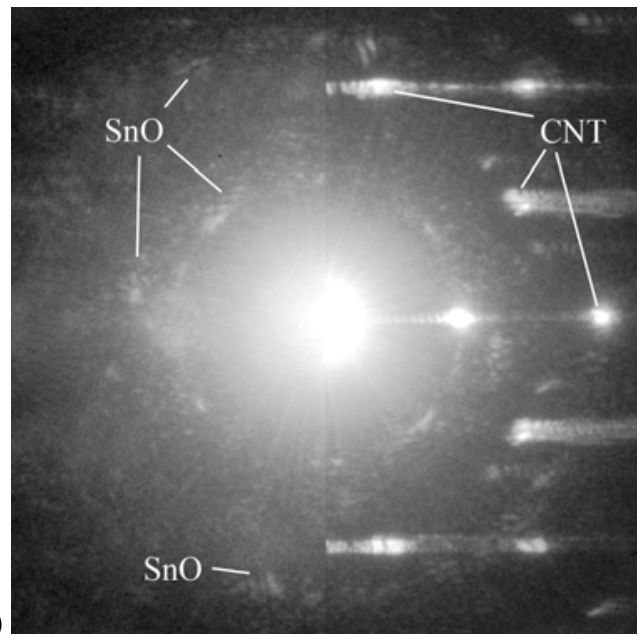

FIG.1. Experimental not optimized TEM image of a) a gold cluster on a carbon support film and c) a $\mathrm{SnO}$ nanoparticle attached to a carbon nanotube. b) Energy-filtered $(8 \mathrm{eV})$ electron diffraction pattern of the gold cluster. The carbon support film has been subtracted by using a diffraction pattern taken at an empty area of the support film. d) Electron diffraction pattern of the $\mathrm{SnO}$ cluster attached to a multi-walled carbon nanotube. The nanotube gives rise to strong ordered spots in the diffraction pattern (right) which can be removed (left) to isolate the diffuse scattering from the $\mathrm{SnO}$ cluster. The resulting diffraction patterns are used in an iterative algorithm to reconstruct the cluster at high resolution without the use of other images. 